\title{
Interruptions of Fictive Scratch Motor Rhythms by Activation of Cutaneous Flexion Reflex Afferents in the Turtle
}

\author{
Scott N. Currie and Paul S. G. Stein \\ Department of Biology, Washington University, St. Louis, Missouri 63130
}

\begin{abstract}
A low-spinal immobilized turtle displays a fictive scratch reflex in hindlimb muscle nerves in response to mechanical stimulation of specific regions of the shell (Robertson et al., 1985). There are 3 forms of the scratch reflex: the rostral, the pocket, and the caudal; each exhibits rhythmic activation of hindlimb motor neurons. Cutaneous stimulation of the distal hindlimb elicits a fictive flexion reflex that exhibits tonic excitation of hip protractor (flexor) motor neurons and tonic inhibition of knee extensor motor neurons (Stein et al., 1982).

In the present study, we describe the motor pattern blends that resulted from transient activation of either the ipsilateral or the contralateral flexion reflex pathway during ongoing scratch motor patterns. Two types of blends were observed: (1) insertions of a flexion reflex synergy into an interrupted scratch cycle and (2) deletions of parts of a scratch cycle. Associated with each type of motor pattern blend was a permanent reset of the ongoing scratch rhythm. The sign of the reset (phase-advance or phase-delay) could be predicted for all forms of the scratch based on the location of the foot stimulus (ipsi- or contralateral) and its timing relative to the hip protractor/retractor cycle. The timing of knee extensor activity within the hip cycle is different for each form of the scratch (Robertson et al., 1985); thus, the sign of the reset cannot be predicted from the timing of the stimulus relative to the knee extensor cycle. These results indicate the importance of the hip rhythm in determining the overall timing of the scratch reflex. In addition, our data support the hypothesis that cutaneous flexion reflex pathways have access to the central rhythm generating circuitry for scratch motor patterns.
\end{abstract}

The turtle hindlimb participates in the motor behaviors of flexion reflex and the 3 forms of the scratch reflex (Stein et al., 1982, 1986a, b; Stein, 1983, 1984; Mortin et al., 1985; Robertson et al., 1985; Robertson, 1986; Robertson and Stein, 1988). The turtle spinal cord, in the absence of movement-related input, selects and generates the organized pattern of motor neuron discharge underlying each of these behaviors. During the flexion reflex, there is excitation of hip protractor motor neurons and inhibition of knee extensor motor neurons. During each of the forms of the scratch reflex, these motor neurons are rhythmically activated. Intrinsic to the spinal cord is the property of selection: for each particular sensory input, a distinct motor output is produced.

Received Mar. 24, 1988; revised July 1, 1988; accepted July 26, 1988.

This research was supported by NIH Postdoctoral Fellowship NS-07850 (to S.N.C.) and NIH Grant NS-15049 (to P.S.G.S.)

Correspondence should be addressed to Dr. Scott N. Currie, Department of Biology, Box 1137, Washington University, St. Louis, MO 63130.

Copyright (c) 1989 Society for Neuroscience $0270-6474 / 89 / 020488-09 \$ 02.00 / 0$
The turtle spinal cord exhibits an elementary form of behavioral choice (Stein et al., 1986a). We demonstrated this using a paradigm of continuous simultaneous activation of 2 cutaneous sites: one in the rostral scratch receptive field and another in the caudal scratch receptive ficld. One of the spinal cord's responses to this paradigm is the construction of motor pattern blends: motor pattern combinations exhibiting characteristics of each of the 2 possible motor output patterns. We observed 2 types of blends: switches and hybrids. During a switch, the spinal cord produces a motor output exhibiting one or more cycles of one motor pattern followed without interruption with one or more cycles of the alternate motor pattern. During a hybrid, the spinal cord produces a motor pattern in which each of several succesive cycles exhibits characteristics of both motor patterns. These blends are observed both with electromyographic (EMG) techniques in a spinal preparation with a moving limb and with electroneurographic (ENG) techniques in a spinal preparation with immobilized muscles. The observations in the immobilized preparation demonstrate that blend formation during behavioral choice is dependent neither upon supraspinal structures nor upon movement-related sensory feedback; blend production is thereforc an intrinsic characteristic of spinal cord neural circuitry.

The present paper describes experiments that examined a different class of behavioral choice, that exhibited when flexion reflex was activated during an ongoing scratch motor rhythm. Flexion reflex was activated by a transient stimulus applied to the dorsum of the foot, either a mechanical tap (Stein et al., 1982; Robertson, 1986; Robertson and Stein, 1988) or a brief train of electrical pulses (Currie and Stein, 1987a, b). The motor response to this stimulus lasted several seconds. The scratch motor rhythm can be continuously produced by the spinal cord for 10-30 sec in response to maintained stimulation of a site in a scratch receptive field. We therefore forced the spinal cord to make a behavioral choice by stimulating the dorsum of the foot in the middle of an ongoing scratch response. Thus, for the several seconds after the flexion stimulus, the spinal cord selected among a flexion motor pattern, a scratch pattern, or some combination of patterns. This paper reports the characteristics of the motor pattern blends in response to such simultaneous stimulation. These results have previously been presented in abstract (Currie and Stein, 1987a, b).

\section{Materials and Methods}

Adult red-eared turtles $(n=38)$, Pseudemys scripta elegans, weight 257 $663 \mathrm{gm}$, were placed in crushed ice for $1 \mathrm{hr}$ prior to surgery to induce hypothermic analgesia (Melby and Altman, 1974; Maxwell, 1979) and were maintained partially immersed in crushed ice during all surgical procedures. The first surgical procedure was complete transection of the spinal cord just caudal to the forelimb enlargement, between the second 
dorsal (D2 = second postcervical) and third dorsal (D3 = third postcervical) spinal segments. A triangular section of the dorsal carapace was then removed over one or both hindlimbs to allow access to hindlimb muscle nerves.

ENG recordings. Several hindlimb muscle nerves were regularly prepared for ENG recording, including IT-KE, AM-KE, FT-KE, VP-HP, and HR-KF. These nerves were described previously (Stein and Grossman, 1980; Stein et al., 1982; Robertson et al., 1985). IT-KE, AM-KE, and FT-KE innervate knee extensor muscles of the triceps femoris group (Walker, 1973): iliotibialis, ambiens, and femorotibialis, respectively. VP-HP innervates puboischiofemoralis internus, pars anteroventralis, a hip protractor muscle. HR-KF innervates several muscles, including the bifunctional hip retractor, knee flexor muscles of the flexor tibialis group. All of these nerves except HR-KF innervate single muscles and so contain axons of single motor pools. Each nerve was freed from surrounding tissues, tied with surgical thread near its muscle insertion, and cut distal to the tie. In approximately half of our experiments, we only prepared hindlimb nerves on the side ipsilateral to the stimulation sites utilized for scratch reflexes $(n=20)$; in the remaining experiments, we also prepared the contralateral VP-HP nerve $(n=18)$ in order to have a monitor for the contralateral flexion reflex

After surgery was complete, the preparation was allowed to warm up to room temperature and was immobilized with an intramuscular injection of Flaxedil (gallamine triethiodide) at a dose of $6 \mathrm{mg} / \mathrm{kg}$ body weight. The trachea was intubated, and artificial respiration was used throughout the experiment. The skin was kept moist with turtle saline. A ring of warm dental wax was formed around the hole(s) in the dorsal carapace over the dissected hindlimb nerves, allowed to cool and harden, and glued in place with Eastman 910 adhesive. The dissected hindlimb nerves were then strung out for recording by securing their attached threads to the lip of the wax ring. Bipolar hook electrodes $(0.004$-inchdiameter silver) were used to record from the nerves in a pool of mineral oil. Data was stored along with a voice channel and stimulus marker on an 8-channel FM tape recorder for later analysis and filming.

Stimulation of scratch and flexion reflexes. We evoked fictive scratch reflexes by gentle mechanical stimulation of the shell surface with a firepolished glass probe. Robertson et al. (1985) have previously described 3 forms of the fictive scratch reflex in the turtle: the rostral scratch, pocket scratch, and caudal scratch. All 3 forms display a rhythmic alternation of hip protractor (flexor) and hip retractor (extensor) nerve activity (Fig. 1). Different scratch forms are discriminated based on the timing of the monoarticular knee extensor, FT-KE, within the hip protractor/retractor cycle. Stimulation of the shell bridge elicits a rostral scratch motor pattern, in which FT-KE is active during the latter portion of VP-HP activity. Stimulation in the shell pocket elicits a pocket scratch motor pattern, in which FT-KE is active during HR-KF activity. Stimulation of sites on the anal plate, near the tail, elicits a caudal scratch motor pattern, in which FT-KE is active after HR-KF activity and before VP-HP activity. The specific shell stimulation sites we utilized are described by Mortin et al. (1985).

We elicited flexion reflexes by brief mechanical or electrical stimulation of the dorsal cutaneous surface of the foot (Fig. 1). Flexion reflex exhibits a multisecond tonic activation of hip protractor (VP-HP) motoneurons and a simultaneous inhibition of knee extensor motoneurons (Stein et al., 1982; Robertson, 1986; Robertson and Stein, 1988). This parallel excitation of hip protractor motor pools and inhibition of knee extensor motor pools constitutes a portion of the flexion reflex synergy.

Mechanical foot stimulation was achieved by tapping the dorsum of the foot with the smooth, rounded tip of a metal probe ( $2 \mathrm{~mm}$ diameter) that was attached to a hand-held strain gauge (Grass FT.03). The output of the force transducer was used as a stimulus marker. The peak force generated by a foot tap ranged between 5 and $11 \mathrm{~N}$ (newtons) and the tap rise time (time from tap onset to peak force) ranged from 5 to 12 msec.

Electrical foot stimulation was produced by gold-plated disk electrodes (Grass, $1 \mathrm{~cm}$ diameter) that were covered on their bottom surfaces with electrically conductive cream and placed about $1 \mathrm{~cm}$ apart on the dorsal surface of the foot. We used rectangular pulse stimulation delivered singly or in brief trains through a stimulus isolation unit. The threshold voltage required to elicit a just detectable flexion reflex discharge in the VP-HP nerve was determined separately for single pulse and train stimuli at the start of each experiment. The intensities of subsequent stimuli were noted as multiples of threshold. We obtained most of our data using 50 or $100 \mathrm{msec}$ trains of $100 \mathrm{~Hz}$ pulses $(6$ or 11 pulses, 1 msec pulse duration) at voltages of $2 \mathrm{~T}-5 \mathrm{~T}$. The maximum



Figure 1. Diagram illustrating the fictive scratch and flexion motor patterns recorded from 5 hindlimb muscle nerves in response to cutaneous stimulation of different regions of the shell and hindlimb in the immobilized turtle with a complete spinal cord transection. IT-KE, AM$\mathrm{KE}$, and FT-KE all innervate knee extensor muscles. VP-HP innervates a hip protractor muscle and HR-KF innervates bifunctional hip retractor/knee flexor muscles (see Materials and Methods). All 3 forms of the scratch reflex display a rhythmic alternation between hip protractor (flexor) and hip retractor (extensor) activity. Scratch forms are discriminated based on the timing of the monoarticular knee extensor, FT-KE, within the hip protractor/retractor cycle. Gentle mechanical stimulation of the shell bridge elicits a rostral scratch motor pattern, in which FT$\mathrm{KE}$ is active during the latter portion of VP-HP activity. Stimulation in the shell pocket elicits a pocket scratch motor pattern, in which FT$\mathrm{KE}$ is active during HR-KF activity. Stimulation of the anal plate, near the tail, elicits a caudal scratch motor pattern, in which FT-KE is active after HR-KF and before VP-HP. A mechanical tap or brief electrical stimulus to the skin on the dorsum of the foot elicits a flexion reflex, characterized by a strong, tonic activation of the VP-HP nerve. The specific stimulus sites referred to in the text are shown in Mortin et al (1985). Turtle diagram adapted from Bojanus (1819).

current passed through the skin ranged from 0.8 to $2.2 \mathrm{~mA}$, as calculated from the skin resistance and stimulus voltage. We measured skin resistance by connecting the inputs of an ohm-meter to the 2 disk electrodes after they had been placed on the foot.

Interruption of scratch reflexes with foot stimulation. Scratch episodes usually began with a short cycle period that progressively lengthened as the motor pattern gradually slowed to a stop. This continuous lengthening of cycle period, especially apparent in short scratch sequences, made it difficult to assess experimental perturbations of the scratch rhythm. We elicited long scratch sequences ( $10-20$ cycles) by maintained mechanical stimulation of a given site within the rostral, pocket, or caudal receptive field. Long scratches, after an initial slowing, usually exhibited several cycles with a fairly constant period before slowing to a stop. We tried to deliver foot stimuli near the middle of this constantperiod region of the scratch, so that any rhythm-resetting effect could be clearly visualized. In cases where cycle period lengthened continuously throughout the scratch sequence, we could still analyze perturbations of the rhythm by aligning the prestimulus portion of the perturbed scratch with a control scratch from the same preparation (Fig. 6).

Illustration of scratch resets. We have drawn lines on our recordings to indicate resets of the scratch rhythm. The vertical lines before foot stimulation indicate the onset of VP-HP nerve activity. The vertical line segments after foot stimulation indicate the expected onset of VPHP activity had no foot stimulation occurred (Figs. 2, 3, 5). The diagonal line segments following foot stimulation indicate the change in VP-HP timing caused by the foot stimulus. VP-HP onset was chosen as our reference point in the scratch cycle because flexion and crossed inhibitory reflexes both act primarily on the VP-HP motor pool and because VP-HP is one of the first motor pools to become active in all 3 scratch forms. In Figure 6, we have drawn lines from the VP-HP onset in the 


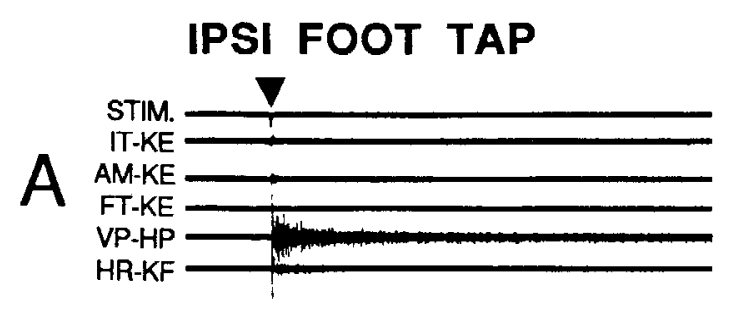

ROSTRAL SCRATCH INTERRUPTED BY IPSI FOOT TAP

$\mathrm{B}$



IPSI FOOT TRAIN (4T)
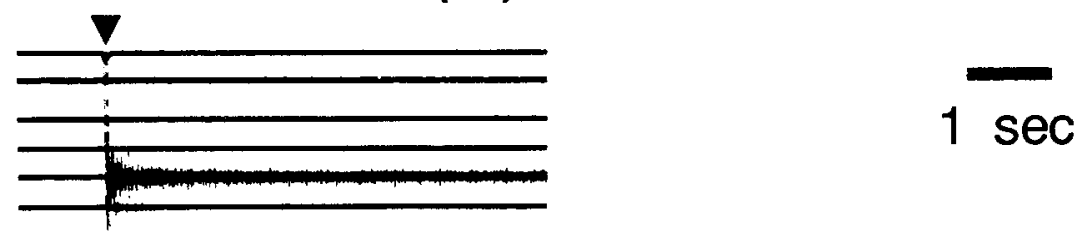

ROSTRAL SCRATCH INTERRUPTED BY IPSI FOOT TRAIN (4T)

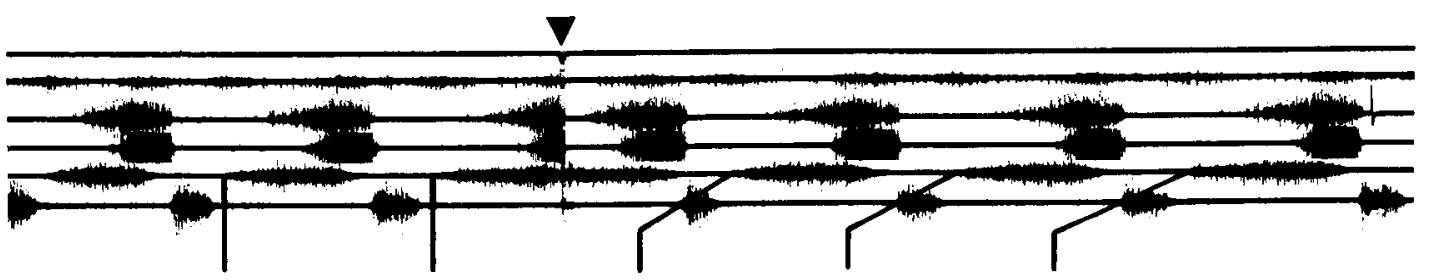

Figure 2. Stimulation of ipsilatcral flexion reflex during an ongoing scratch motor pattern can permancntly resct the scratch rhythm. Foot stimulation is indicated by inverted triangles. A, Mechanical tap to the ipsilateral dorsum of foot elicited a fictive flexion reflex in the hip protractor (flexor) nerve VP-HP in the resting preparation. $B$, A similar mechanical stimulus delivered to ipsilateral foot during the early hip protractor phase of a rostral scratch caused a permanent phase-delay reset of the scratch rhythm. $C$, An electrical stimulus delivered to the ipsilateral dorsum of foot (6 pulses, $100 \mathrm{~Hz}, 4 \times$ threshold) elicited a flexion reflex. $D$, A similar electrical stimulus delivered during late hip protractor phase (after FT-KE activation began) caused a phase-delay reset of the scratch rhythm.

control scratch sequence to the corresponding VP-HP onset in the perturbed sequence.

Calculation of percent phase shifts. To calculate the percentage phaseshift values presented in Tables 1 and 2 , we examined scratch sequences where (1) the foot stimulus reset the scratch rhythm and (2) the cycle periods immediately prior to and following the perturbed scratch cycle were the same length (i.e., cycle period was constant at the point in the scratch sequence when the foot stimulus was applied). We calculated the ratio of the perturbed cycle period to the prestimulus cycle period; values greater than 1.0 indicate a phase delay, while values less than 1.0 indicate a phase advance. This ratio was then multiplied by 100 , subtracted from $100 \%$, and expressed as an absolute number to obtain the final percentage phase-shift value.

\section{Results}

\section{Interaction of ipsilateral flexion reflex and scratch motor} patterns

Activation of an ipsilateral flexion reflex by brief stimulation of the dorsum of the foot could permanently reset the rhythm of an ongoing scratch motor pattern. A mechanical tap to the dor- sum of foot in a resting preparation produced a flexion reflex (Fig. $2 A$ ) characterized by the robust activation of the hip protractor nerve, VP-HP. Tapping the foot during the early hip protractor phase of an ongoing rostral scratch (prior to FT-KE activation) lengthened the VP-HP burst, inhibited activity in all 3 knee extensors, and caused a permanent phase-delay reset of the scratch rhythm (Fig. $2 B$ ). Electrical stimulation of the dorsum of foot in the same preparation also produced a normal flexion reflex (Fig. 2C). Delivery of this stimulus during the late hip protractor phase of the rostral scratch (during FT-KE activity) lengthened the VP-HP burst, temporarily inhibited knee extensor activity, and again caused a phase-delay reset of the scratch (Fig. 2D). AM-KE and FT-KE reinitiated their bursts following the foot stimulus and did so in the proper rostral scratch sequence of AM-KE onset preceding FT-KE onset.

The responses in Figure 2, $B, D$, both displayed an insertion of the flexor reflex motor pattern (excitation of hip protractors and inhibition of knee extensors) that briefly interrupted and 


\section{IPSILATERAL FOOT STIMULATION}

\section{A ROSTRAL - DURING HP}

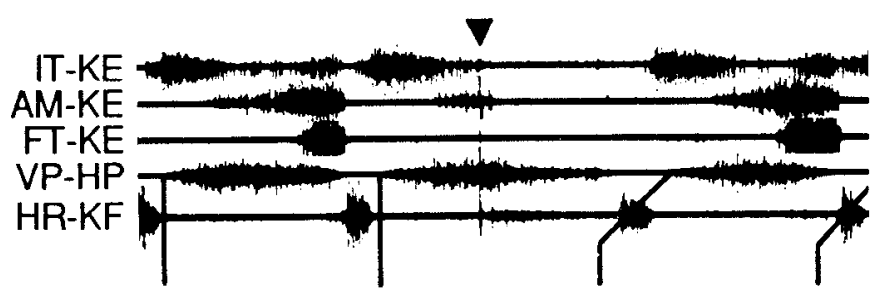

C POCKET - DURING HP

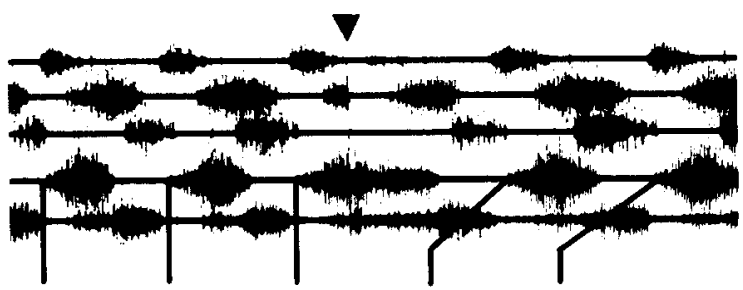

E CAUDAL - DURING HP

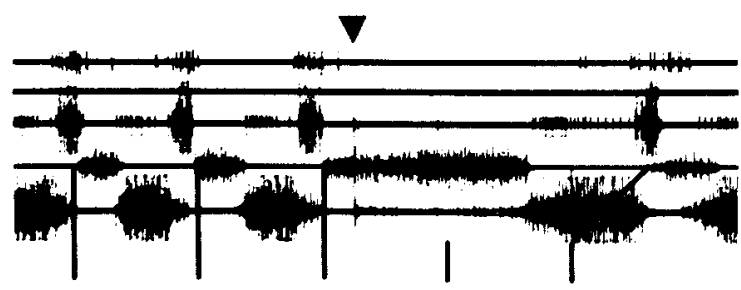

B ROSTRAL - DURING HR

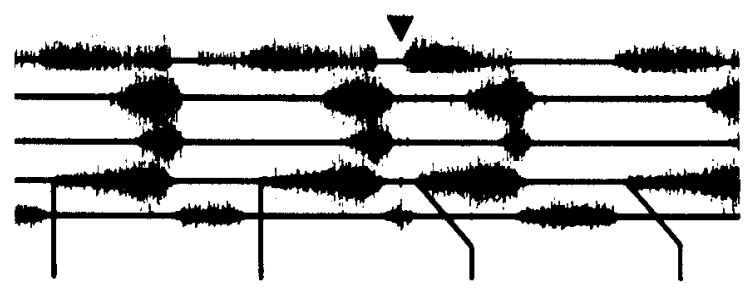

D POCKET - DURING HR

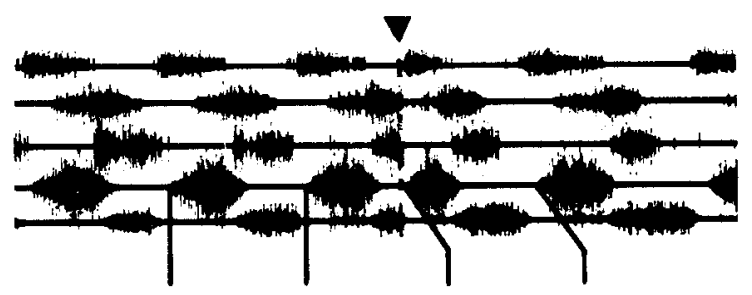

F CAUDAL - DURING HR

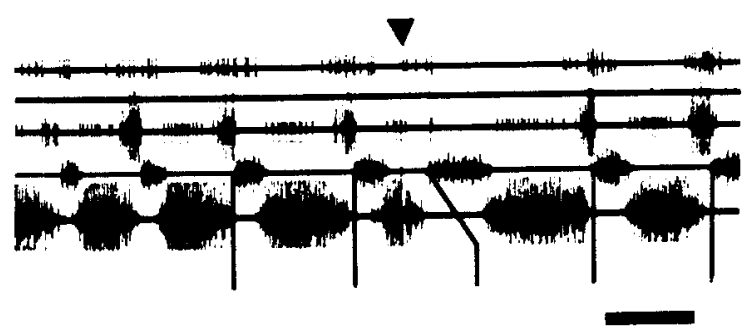

$1 \mathrm{sec}$

Figure 3. Phase-dependent effects of ipsilateral foot stimuli on fictive scratch rhythms. Stimulation of the ipsilateral foot during the hip protractor (HP) phase of a rostral, pocket, or caudal scratch $(A, C, E)$ lengthened the hip protractor (VP-HP) burst and produced a phase-delay reset of the scratch rhythm. Stimulation during the hip retractor (HR) phase $(B, D, F)$ shortened the activity of the hip retractor (HR-KF) motor pool and produced a phase-advance reset of the scratch rhythm. Foot stimuli are indicated by inverted triangles. Mechanical foot stimulation was used in $A-C, E, F$; electrical stimulation (6 pulses, $100 \mathrm{~Hz}, 2 \times$ threshold) was used in $D$.

reset the ongoing rostral scratch motor pattern. This insertion was especially clear since the rostral scratch motor pattern is an example of a "mixed flexor/extensor synergy": The knee extensors are coactive with the hip (flexors) protractors. The overall motor response was therefore an example of a motor blend since it displayed both a rostral scratch pattern and a flexion reflex pattern. The insertion could also be viewed as a double switch in which the motor pattern changed from a rostral scratch to a flexion reflex and then changed back again to a rostral scratch in the same cycle of hip protraction and retraction. The interruptions observed here do not qualify as a hybrid according to our blend classification scheme (Mortin et al., 1985; Robertson et al., 1985; Stein et al., 1986a, b); classification as a hybrid requires the expression of both motor patterns for each of several successive cycles.

Stimulation of ipsilateral foot during the hip protractor phase of each of the 3 scratch forms caused the insertion of a flexion reflex into the scratch motor pattern (Fig. 3, $A, C, E$ ). This insertion lengthened the hip protractor (VP-HP) burst, inhibited or delayed knee extensor and hip retractor activity, and could produce a phase delay of the scratch rhythm. Delivery of the foot stimulus during the hip protractor phase of the caudal scratch (Fig. 3F) lengthened the VP-HP burst to such an extent that the scratch skipped a cycle and produced a phase delay of the subsequent scratch rhythm.

Stimulation of ipsilateral foot during the hip retractor (extensor) phase of a scratch terminated the HR-KF burst and caused a phase advance of the scratch rhythm (Fig. 3, $B, D, F$ ). The only expression of a flexion reflex synergy during the hip retractor phase of the rostral scratch (Fig. $3 B$ ) was the inhibition of HR-KF (hip extensor) activity. During the pocket and caudal scratches (Fig. 3, D,F), there was also an inhibition of the knee extensor discharge. The phase-advanced VP-HP bursts that followed stimulation in the rostral and pocket scratches exhibited 

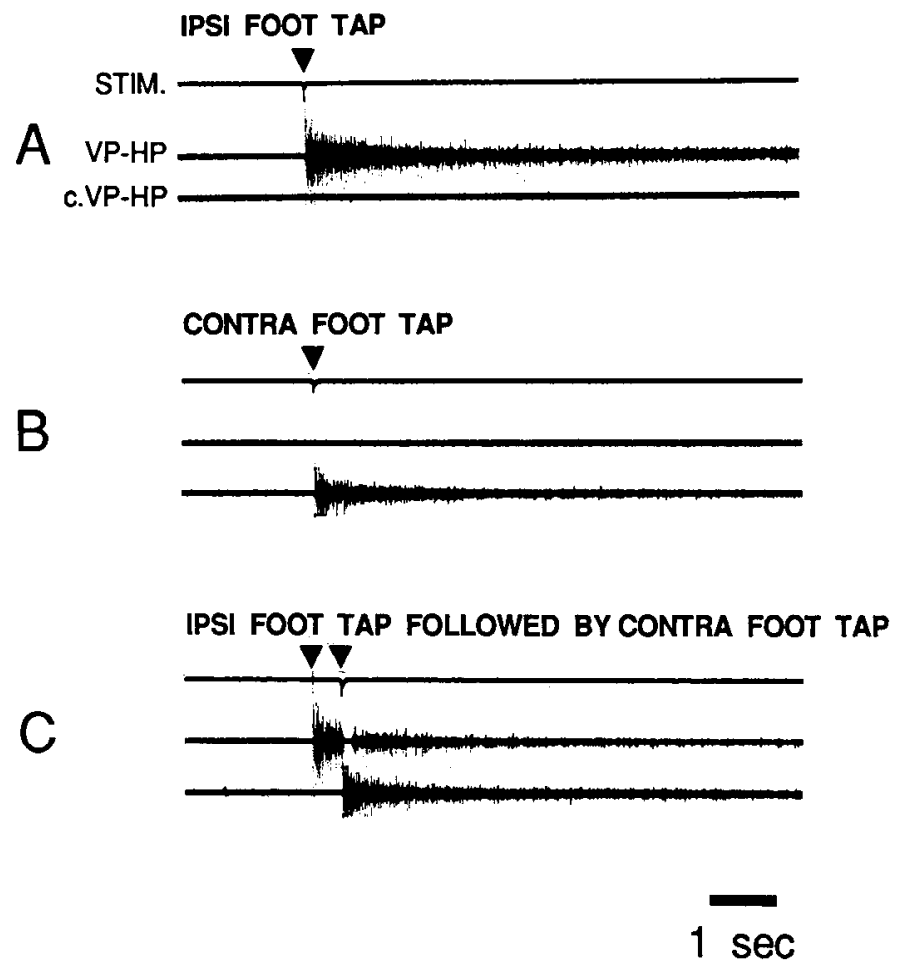

Figure 4. Crossed inhibition of hip protractor (flexor) nerve activity during contralateral flexion reflex. $A$, Mechanical tap to the ipsilateral dorsum of foot produced a normal flexion reflex in the ipsilateral VPHP nerve. $B$, Tapping the contralateral dorsum of foot produced a flexion reflex in the contralateral VP-HP nerve (c. VP-HP). C, Tapping ipsilateral and contralateral dorsum of feet in quick succession revealed a crossed inhibitory effect of the contralateral stimulus on ipsilateral VP-HP activity.

the fusiform shape and small-to-large recruitment order characteristic of those forms, rather than the sudden onset of a flexion reflex, in which large and small motor units were all simultaneously activated. These features indicated that the response to the foot stimulation elicited a deletion of the latter portion of the HR-KF phase of the normal scratch motor pattern. The phase-advanced caudal scratch (Fig. $3 F$ ) compensated for the perturbation by lengthening the VP-HP and HR-KF bursts in the first poststimulus cycle, bringing the motor pattern back into alignment with the timing that would have been expressed had no foot stimulus been given. Such cases of compensation and realignment with the prestimulus rhythm could occur in perturbations of all 3 scratch forms but were especially common in the caudal scratch.

In Table 1, we show the sign and magnitude of the phase shifts for all of the cases where ipsilateral foot stimulation reset the scratch rhythm. The magnitude of the resets was highly variable, depending on such factors as the strength and timing of the foot stimulus and the briskness of the scratch. In contrast, the sign of the reset (phase advance or delay) exhibited a consistent phase dependence. Ipsilateral foot stimulation during the hip protractor phase always evoked a phase delay; stimulation during the hip retractor phase always evoked a phase advance. Thus, the sign of the reset produced by an ipsilateral foot stimulus could be predicted for any scratch form based solely on the timing of the foot stimulus relative to the hip protractor/retractor cycle.

\section{Crossed inhibition during flexion reflex}

Stimulation of flexion reflex in the contralateral hindlimb caused a strong crossed inhibition of ipsilateral hip protractor (flexor) nerve activity (Fig. 4). The control flexion reflex resulting from a tap to the ipsilateral dorsum of foot (Fig. $4 A$ ) and the control contralateral flexion reflex resulting from a contralateral foot tap (Fig. $4 B$ ) each displayed multisecond activation of the VPHP nerve in the limb receiving the tap. Tapping the ipsilateral foot first followed rapidly by tapping the contralateral foot revealed a temporary cessation and, therefore, a crossed inhibition of ipsilateral hip protractor nerve discharge in response to activation of the contralateral flexion reflex pathway. We tested 30 turtles for this response; 28 of these exhibited some degree of crossed inhibition. The intensity of the inhibition varied widely among preparations. In some turtles, the diminution of ipsilateral hip protractor activity caused by the contralateral stimulus was barcly perceptible, whilc in others the hip protractor discharge was cut off completely. Electrical train stimulation of the contralateral foot usually produced a stronger crossed inhibition than a mechanical tap.

Some turtles also exhibited a weak crossed extension discharge in the hip retractor nerve during contralateral foot stimulation. Occasionally, this effect could be elicited by a brief mechanical tap or electrical train stimulus to the contralateral foot. Usually, more intense and maintained stimulation, e.g.,

Table 1. Pooled data showing the sign and magnitude of phase shifts resulting from ipsilateral foot stimulation at different times in the hip cycle for each of the 3 scratch forms

\begin{tabular}{llllll} 
& \multicolumn{2}{l}{ Stimulation during HP } & & Stimulation during HR \\
\cline { 2 - 3 } Reflex & $\begin{array}{l}\text { Phase } \\
\text { advance }\end{array}$ & Phase delay & & Phase advance & $\begin{array}{l}\text { Phase } \\
\text { delay }\end{array}$ \\
\hline Rostral & $0 / 19$ & $19 / 19$ & $9 / 9$ & $0 / 9$ \\
& & $(11-199 \%, \bar{x}=61.9 \%)$ & & $(10-27 \%, \bar{x}=19.2 \%)$ & \\
Pocket & $0 / 3$ & $3 / 3$ & $8 / 8$ & $0 / 8$ \\
& & $(10-67 \%, \bar{x}=34.7 \%)$ & & $(10-52 \%, \bar{x}=30.1 \%)$ & \\
Caudal & $0 / 4$ & $4 / 4$ & & $13 / 13$ & $0 / 13$ \\
& & $(55-148 \%, \bar{x}=113.2 \%)$ & & $(8-59 \%, \bar{x}=23.8 \%)$ &
\end{tabular}

The denominator in each ratio is the total number of flexion-scratch interactions observed for a given combination of scratch form and stimulus timing; the numerator is the number of times we observed either a phase advance or phase delay within that group. Also indicated in parentheses are the range and mean $(\bar{x})$ of the percentage phase shift for each group. 


\section{CONTRALATERAL FOOT STIMULATION}

\section{A ROSTRAL - DURING HP}



\section{POCKET - DURING HP}

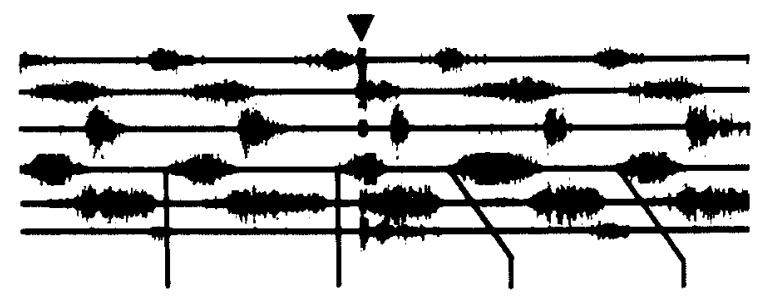

B ROSTRAL - DURING HR

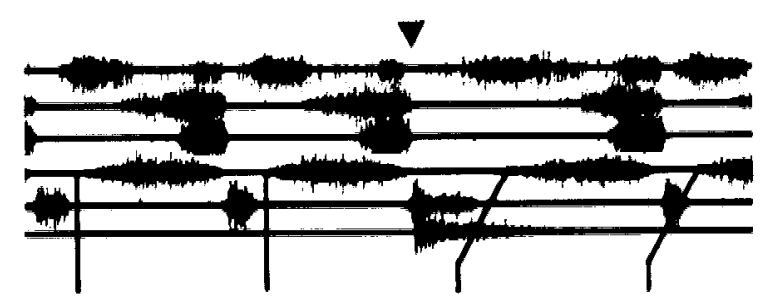

D POCKET - DURING HR

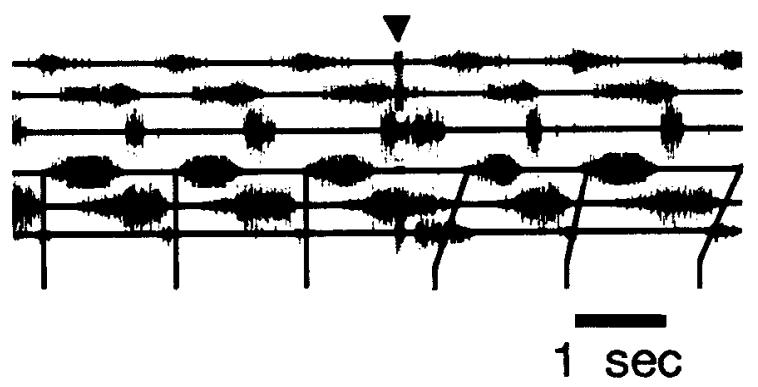

Figure 5. Phase-dependent effects of contralateral foot stimuli on fictive scratch rhythms. Stimulation of the contralateral foot during the HP phase of a rostral or pocket scratch $(A, C)$ shortened the activity of the VP-HP motor pool and produced a phase advance of the scratch rhythm. Contralateral foot stimulation applied during the HR phase $(B, D)$ lengthened the activity of the HR-KF motor pool and produced a phase delay of the scratch rhythm. Foot stimuli are indicated by inverted triangles. $c . V P$ - $H P$, contralateral VP-HP neurogram. Mechanical foot stimuli wcre used in $A$ and $B$; electrical stimuli (11 pulses, $100 \mathrm{~Hz}, 5 \times$ threshold) were used in $C$ and $D$.

pinching the skin on the dorsum of the foot with forceps, was required to produce crossed extension.

\section{Interaction of contralateral flexion reflex and scratch motor patterns}

Crossed inhibition of hip protractor nerve discharge was also expressed during interactions of contralateral flexion reflex with ipsilateral scratch motor patterns. Stimulation of contralateral foot during the hip protractor phase of a rostral or pocket scratch reflex shortened the hip protractor burst, caused a premature activition of knee extensor and hip retractor motor pools, and produced a phase advance of the scratch rhythm (Fig. 5, $A, C$ ). These are examples of deletion of a portion of the scratch cycle. Stimulation of contralateral foot during the hip retractor (ex- tensor) phase of a rostral or pocket scratch lengthened the hip retractor burst and caused a phase delay of the scratch rhythm (Fig. 5, B, D). These are examples of an insertion of extensor activity into the scratch cycle. For both the rostral and pocket scratch, the foot stimulation affected burst length and cycle timing, but preserved the motor pool synergies characteristic of the ongoing scratch form. Thus, the stimulus extended the activity of the HR-KF motor pool alone during the rostral scratch (Fig. $5 B$ ) but lengthened both the HR-KF and coactive FT-KE bursts in the pocket scratch (Fig. 5D). We were unable to demonstrate clear perturbations of caudal scratch motor rhythms with contralateral foot stimulation. In some cases, stimulation appeared to disturb the intracycle motor pattern; however, the effect tended to be weak.

Table 2. Pooled data showing the sign and magnitude of phase shifts resulting from contralateral foot stimulation at different times in the hip cycle for the rostral and pocket forms of the scratch

\begin{tabular}{|c|c|c|c|c|}
\hline \multirow[b]{2}{*}{ Reflex } & \multicolumn{2}{|l|}{ Stimulation during $\mathbf{H P}$} & \multicolumn{2}{|c|}{ Stimulation during $\mathrm{HR}$} \\
\hline & Phase advance & $\begin{array}{l}\text { Phase } \\
\text { delay }\end{array}$ & $\begin{array}{l}\text { Phase } \\
\text { advance }\end{array}$ & Phase delay \\
\hline Rostral & $\begin{array}{l}10 / 10 \\
(13-32 \%, \bar{x}=19.8 \%)\end{array}$ & $0 / 10$ & $0 / 4$ & $\begin{array}{l}4 / 4 \\
(20-84 \%, \tilde{x}=32.2 \%)\end{array}$ \\
\hline Pocket & $\begin{array}{l}3 / 3 \\
(8-32 \%, \bar{x}=17.3 \%)\end{array}$ & $0 / 3$ & $0 / 6$ & $\begin{array}{l}6 / 6 \\
(26-142 \%, x=53.3 \%)\end{array}$ \\
\hline
\end{tabular}






ROSTRAL SCRATCH

B

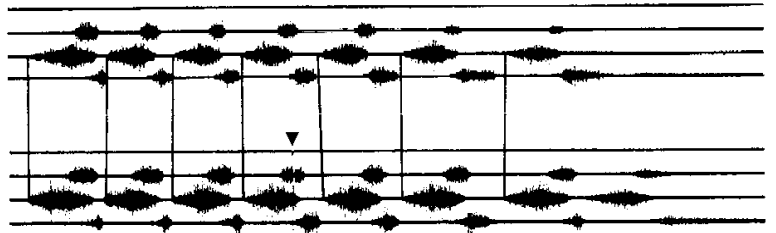

ROSTRAL SCRATCH INTERRUPTED BY IPSI FOOT PULSE (3T)

$\mathrm{D}$

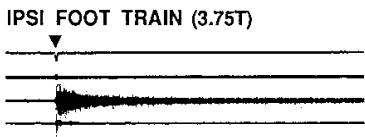

$1 \mathrm{sec}$

ROSTRAL SCRATCH

E

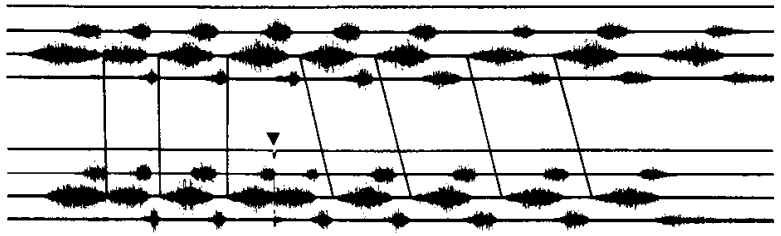

ROSTRAL SCRATCH INTERRUPTED BY IPSI FOOT TRAIN (3.75T)

Figure 6. The foot stimulation intensity required to reset a scratch rhythm was higher than the intensity rcquircd for a flexion reflex. Foot stimuli are indicated by inverted triangles. $A$, Single pulse electrical stimulus to the ipsilateral dorsum of foot $(1 \mathrm{msec}, 3 \times$ threshold $)$ in the resting preparation was adequate to evoke a weak flexion reflex. $B$, A control rostral scratch. $C$, The $3 \mathrm{~T}$ pulse to ipsilateral dorsum of foot during an ongoing rostral scratch produced a brief inhibition of FT-KE activity but no significant perturbation of the scratch rhythm. $D$, Brief electrical train to the ipsilateral dorsum of foot $(6$ pulses, $100 \mathrm{~Hz}, 3.75$ $\times$ threshold) produced a strong flexion reflex. $E$, Control rostral scratch. $F$, The $3.75 \mathrm{~T}$ train to ipsilateral dorsum of foot caused a phase-delay reset relative to the rostral scratch control. Lines are drawn between the VP-HP onsets in $B$ and $C$ and in $E$ and $F$ to aid in comparisons of cycle timing.

The effects produced by contralateral foot stimuli on hip motor pool activities and on the scratch rhythm were opposite the effects produced by ipsilateral foot stimuli. The consistency of this phase dependence is shown in Table 2. Stimulation of contralateral foot during the hip protractor phase always produced a phase advance; stimulation during the hip retractor phase always produced a phase delay. Thus, the sign of the reset produced by a contralateral foot stimulus could be predicted for a rostral or pocket scratch from the timing of the foot stimulus relative to the hip cycle.

\section{Stimulus threshold for a flexion reflex is lower than the stimulus threshold for the resetting of the scratch rhythm}

Single pulses to the ipsilateral dorsum of foot at voltages greater than $2 \mathrm{~T}$ evoked flexion reflexes in a resting preparation but were usually too weak to perturb an ongoing scratch rhythm. To obtain clear scratch resets with electrical stimuli, it was usually necessary to stimulate the foot with a short train of pulses. We compare directly the effects of single-pulse and train stimulation of the ipsilateral foot on rostral scratch motor patterns in Figure 6. Cycle period increased continuously in these scratch bouts; thus, we illustrate the effects of foot stimulation by aligning the interrupted scratches with uninterrupted control scratches from the same preparation.

A single pulse to ipsilateral foot produced a weak flexion reflex while the preparation was at rest (Fig. 6A); when a stimulus pulse of the same magnitude was delivered during the late hip protractor phase of a rostral scratch, it caused a brief inhibition of knee extensor (FT-KE) activity but had only a minor effect on the rostral scratch rhythm compared with the uninterrupted control scratch (Fig. 6, B, C). In contrast, a train of 6 pulses $(100 \mathrm{~Hz})$ produced a very intense flexion reflex in the resting preparation (Fig. $6 D$ ) and caused a marked phase delay in the rostral scratch rhythm (Fig. $6, E, F$ ). The 3 T single pulse and $3.75 \mathrm{~T}$ train had the same voltage; their intensities relative to threshold were different because trains evoke flexion reflex at a lower voltage threshold than single pulses.

\section{Discussion}

Stimulation of flexion reflex during an ongoing fictive scratch reflex could result in a motor pattern blend and a phase shift in the scratch rhythm. Two classes of motor blend were observed in this behavioral choice paradigm: (1) insertions associated with a phase delay and (2) deletions associated with a phase advance. Insertions occurred in response to stimulation of the ipsilateral foot during hip protractor (flexor) activity and in response to stimulation of the contralateral foot during the hip retractor (extensor) activity. In contrast, deletions occurred in response to stimulation of the ipsilateral foot during hip retractor (extensor) activity and in response to stimulation of the contralateral foot during hip protractor (flexor) activity. Thus, the sign, either an advance or delay, of the phase shift depended upon the time of the cycle of hip protraction and retraction during which stimulation was applied and the location of the foot stimulus (ipsi- or contralateral) relative to the scratch stimulus.

The above generalizations held for both the rostral and the pocket scratches and for the ipsilateral flexion effect on the caudal scratch. Since the timing of the monoarticular knee extensor (FT-KE) is different for each of the forms of the scratch (Fig. 1), it follows that the sign of the phase shift could not be predicted by knowing only the timing of knee extensor activity. For example, stimulation of the ipsilateral flexion reflex during the FT-KE activity of a rostral scratch was associated with a phase delay (Fig. 2D); in contrast, stimulation of the ipsilateral flexion reflex during FT-KE activity of a pocket scratch was associated with a phase advance (Fig. $3 D$ ). The type of phase shift that resulted when a flexion reflex was elicited during activity of the monoarticular knce cxtensor motor neurons therefore depended upon which of the hip motor neuron pools were active when the monoarticular knee extensors were active, i.e., upon the particular form of the scratch that was expressed. Thus, the importance of the phase of the hip cycle in the prediction of the sign of the reset is consistent with the following hypotheses: (1) The timing of the hip motor output is critically related to the timing of the rhythm of the entire limb, and (2) the timing of motor output to more distal musculature is adjusted secondarily according to the hip motor timing. The importance of the hip in the control of the limb rhythm has been emphasized previously in the work of Andersson and Grillner (1981, 1983) on the effects of hip sensory input on the stepping rhythm in the cat.

Our results, that stimulation of a cutaneous flexion reflex may reset the phasing of a hindlimb motor rhythm, contradict con- 
clusions reached by Lennard (1985; Lennaid and Hermanson, 1985 ) in work with the swim rhythm of the turtle. He evoked a swim motor rhythm and actual swimming movements in a single hindlimb with focal electrical stimulation of sites in the dorsolateral funiculus of the spinal cord. He used electrical stim= ulation of a cutaneous nerve to elicit a flexion reflex EMG re= sponse in the hip protractor musculature. He was careful to use a relatively weak electrical stimulus so that the hindlimb did not exhibil an actual withdrawal response to the stimulus. $\mathrm{He}$ correctly reasoned that such an actual withdrawal would elicit muscle spindle reafferent activity; he knew from other experiments that direct stimulation of muscle afferents could reset the swim cycle; he therefore limited his cutaneous nerve stimulation to stimulus strengths that would be adequate to evoke a weak activation of hip flexor motor units and would not be strong enough to cause an actual flexion reflex movement. In general, he used a single electrical pulse to a cutaneous nerve at $2-3 \mathrm{~T}$; he observed transient phase shifts and not permanent phase shifts in response to the stimulus. Our work differs from Lennard's: we worked with a fictive preparation that exhibited the scratch motor rhythm and the flexion reflex in the absence of actual movement. We were therefore not subject to Lennard's constraint of having to use only a weak cutaneous stimulus. When we used a single pulse of electrical stimulation in the fictive preparation, we could get a weak flexion motor response (Fig. 6A). When a single pulse was delivered during an ongoing scratch, it did not reset the scratch rhythm; it only caused a transient inhibition of knee extensor discharge. In our preparation, we could stimulate the foot with a train of electrical pulses that were sufficient to produce a much stronger flexion reflex response (Fig. $6 D$ ). This stimulus, when delivered during an ongoing fictive scratch motor rhythm, was sufficient to generate a permanent reset of the scratch cycle. Thus, our conclusion working with fictive motor rhythms is that a cutaneous stimulus does have access to the scratch timing circuitry and can reset the rhythm. Had Lennard worked with a fictive swim preparation, our prediction is that he could have tested the effects of stronger cutaneous stimulation and would have observed a permanent reset.

Our observations with the turtle are similar to those previously observed in mammals. The contralateral flexion reflex inhibition of an ipsilateral flexion reflex is exhibited by a spinal $\operatorname{dog}$ (Sherrington, 1906) and a spinal cat (Eccles and Sherrington, 1931; Holmqvist, 1961; Jankowska et al., 1967). In addition, Sherrington (1906) observed the interruption of the dog's scratch reflex with a flexion reflex. Modifications in the sign of the phase shift of a scratch cycle according to the timing of a perturbing stimulus, e.g., stimulation of a dorsal root, are observed in the cat (Bcrkinblit et al., 1978). There is an extensive literature on modification in the sign of the phase shift in the step cycle of the cat according to the timing of a perturbing stimulus (Duysens and Pearson, 1976; Duysens, 1977; Forssberg et al., 1977; Duysens and Stein, 1978; see especially Rossignol et al., 1988). Such phase-dependent effects are observed in most biological oscillators (Pavlides, 1973; Stein, 1976).

Our observations with the effects of cutaneous stimulation on the scratch cycle differ from experiments performed on spinal cats during stepping that demonstrate phase-dependent refiex reversal: a stimulus presented during flexion elicits a more intense flexion response and the same stimulus presented during extension elicits a more intense extension response (Forssberg et al., 1977; Rossignol et al., 1988). Reflex reversal in the cat is obscrved fờ soñine, būt not all, cutaneous reflexcs. The behavioral significance of this reversal is that the locomoting cat displays a response that is adaptive toward maintaining a proper balance. In the turtle scratch, reflex reversal may not be required; we did not observe reflex reversal in the present set of experinonents:

We have shown that stimulation of cutaneous flexion reflex afferents in the turtle can permanently reset the rhythm of fictive scratch motor patterns. We therefore infer that cutaneous flexion reflex pathways have access to the rhythm-generating circuitry for scratch reflexes. A possible mechanism for such access is that some of the interneurons involved in flexion reflex are also directly involved in the production of scratch reflex. Such a mechanism has been proposed for flexion reflexes and stepping in the L-DOPA-treated spinal cat (Jankowska et al, 1967; Lundberg, 1981). At least some of the interneuronal circuitry involved in flexion must differ from that involved in scratch, however. This conclusion is based on the observation that the flexion reflex can interrupt the scratch with the insertion of a flexion synergy and the temporary suppression of a scratch synergy. Future work with direct recordings from interneurons during flexion reflex and the 3 forms of the scratch will provide evidence for these hypotheses.

\section{References}

Andersson, O., and S. Grillner (1981) Peripheral control of the cat's step cycle. I. Phase dependent effects of ramp-movements of the hip during "fictive locomotion." Acta Physiol. Scand. 113: 89-101.

Andersson, O., and S. Grillner (1983) Peripheral control of the cat's step cycle. II Entrainment of the central pattern generators for locomotion by sinusoidal hip movements during "fictive locomotion." Acta Physiol. Scand. 118: 229-239.

Berkinblit, M. B., T. G. Deliagina, A. G. Feldman, I. M. Gelfand, and G. N. Orlovsky (1978) Generation of scratching. II. Nonregular regimes of generation. J. Neurophysiol. 41: 1058-1069.

Bojanus, L. H. (1819) Anatome Testudinis Europaeae, Vilnae, Bojanus [reprinted Society for Study of Amphibians and Reptiles, 1970].

Currie, S., and P. S. G. Stein (1987a) Interaction of flexion and scratch reflexes in the turtle. Neuroscience 22: $\$ 653$.

Currie, S., and P. S. G. Stein (1987b) Interactions between cutaneous flexion reflex pathways and scratch central pattern generators in the turtle. Soc. Neurosci. Abstr. 13: 823 .

Duysens, J. (1977) Fluctuations in sensitivity to rhythm resetting effects during the cat's step cycle. Brain Res. 133: 190-195.

Duysens, J., and K. G. Pearson (1976) The role of cutaneous afferents from the distal hindlimb in the regulation of the step cycle of thalamic cats. Exp. Brain Res. 24: 245-255.

Duysens, J., and R. B. Stein (1978) Reflexes induced by nerve stimulation in walking cats with implanted cuff electrodes. Exp. Brain Res. 32: 213-224.

Eccles, J. C., and C. S. Sherrington (1931) Studies on the flexor reflex. VI. Inhibition. Proc. R. Soc. London [Biol.] 109: 91-113.

Forssberg, H., S. Grillner, and S. Rossignol (1977) Phasic gain control of reflexes from the dorsum of the paw during spinal locomotion. Brain Res. 132: 121-139.

IIolmqvist, B. (1961) Crossed spinal reflex actions cvoked by vollcys in somatic afferents. Acta Physiol. Scand. Suppl. 181 52: 1-66.

Jankowska, E., M. G. M. Jukes, S. Lund, and A. Lundberg (1967) The effect of DOPA on the spinal cord. 5. Reciprocal organization of pathways transmitting excitatory action to alpha motoneurones of flexors and extensors. Acta Physiol. Scand. 70: 369-388.

Lennard, P. R. (1985) Afferent perturbations during "monopodal" swimming movements in the turtle: Phase-dependent cutaneous modulation and proprioceptive resetting of the locomotor rhythm. J. Neurosci. 5: 1434-1445.

Lennard, P. R., and J. W. Hermanson (1985) Central reflex modulation during locomotion. Trends Neurosci. 8: 483-486.

Lundberg, A. (1981) Half-centres revisited. Adv. Physiol. Sci. 1: 155167. 
Maxwell, J. H. (1979) Anesthesia and surgery. In Turtles: Perspectives and Research, M. Harless and H. Morlock, eds., pp. 127-152, Wiley, New York.

Melby, E. C. J., and N. H. Altman (1974) Handbook of Laboratory Animal Science, Vol, 1, CRC, Cleveland

Mortin, L. I., J. Keifer, and P. S. G. Stein (1985) Three forms of the scratch reflex in the spinal turtle: Movement analyses. J. Neurophysiol. 53: 1501-1516.

Pavlidis, T. (1973) Biological Oscillators: Their Mathematical Analysis, Academic, New York.

Robertson, G. A. (1986) Synaptic control of motor neurons in the production of three forms of the fictive scratch in the turtle, Doctoral Dissertation, Washington University in St. Louis.

Robertson, G. A., and Stein, P. S. G. (1988) Synaptic control of hindlimb motoneurones during three forms of the fictive scratch reflex in the turtle. J. Physiol. (Lond.) 404: 101-128.

Robertson, G. A., L. I. Mortin, J. Keifer, and P. S. G. Stein (1985) Three forms of the scratch reflex in the spinal turtle: Central generation of motor patterns. J. Neurophysiol. 53: 1517-1534.

Rossignol, S., J. P. Lund, and T. Drew (1988) The role of sensory inputs in rcgulating patterns of rhythmical movements in higher vertebrates: A comparison between locomotion, respiration, and mastication. In Neural Control of Rhythmic Movements in Vertebrates, A. H. Cohen, S. Rossignol, and S. Grillner, eds., pp. 201-283, Wiley, New York.

Sherrington, C. S. (1906) The Integrative Action of the Nervous System, Yale University Press, New Haven, CT.
Stein, P. S. G. (1976) Mechanis̄nss of interlimb phase control. In Neural Control of Locomotion, R. M. Herman, S. Grillner, P. S. G. Stein, and D. G. Stuart, eds., pp. 465-487, Plenum, New York.

Stein, P. S. G. (1983) The vertebrate scratch reflex. Symp. Soc. Exp. Biol. 37: 383-403.

Stein, P. S. G. (1984) Central pattern generators in the spinal cord. In Handbook of the Spinal Cord, Vols. 2 and 3: Anatomy and Physiology, R. A. Davidoff, ed., pp. 647-672, Dekker ${ }_{3}$ New York.

Stein, P. S. G., and M. L. Grossman (1980) Central program for scratch reflex in turtle. J. Comp. Physiol. 140: 287-294.

Stein, P. S. G., G. A. Robertson, J. Keifer, M. L. Grossman, J. A. Berenbeim, and P. R. Lennard (1982) Motor neuron synaptic potentials during fictive scratch reflex in turtle. J. Comp. Physiol. 146: $401=409$.

Stein, P. S. G., A. W. Camp, G. A. Robertson, and L. I. Mortin (1986a) Blends of rostral and caudal scratch reflex motor patterns elicited by simultaneous stimulation of two sites in the spinal turtle. J. Neurosci. $6 \cdot 2.259-2.266$.

Stein, P. S. G., L. I. Mortin, and G. A. Robertson (1986b) The forms of a task and their blends. In Neurobiology of Vertebrate Locomotion, S. Grillner, P. S. G. Stein, D. G. Stuart, H. Forssberg, and R. M. Herman, eds., pp. 201-216, Macmillan, New York.

Walker, W. F. (1973) The locomotor apparatus of testudines. In $\mathrm{Bi}$ ology of the Reptilia, Vol. 4, C. Gans and T. S. Parsons, eds., pp. 1100 , Academic, New York. 